\title{
Solving Multi-Area Economic Dispatch with Multiple Fuels Using Hybrid Optimization Technique
}

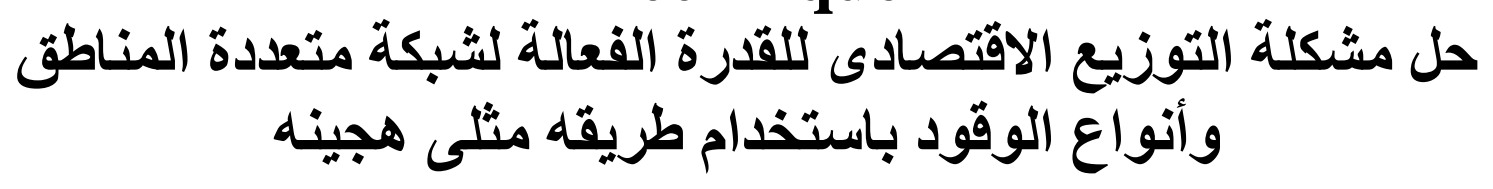

\author{
Ehab E. Elattar \\ Department of Electrical Engineering, Faculty of Engineering, \\ Minoufiya University, Shebin El-Kom, Egypt
}

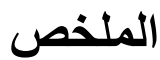

مشكلة التوزيع الاقتصادى للقدرة الفعالة لثبكة متعددة المناطق تهتم بالتوزيع الامثل للقدرة الفعالة لكل المولدات فى التى

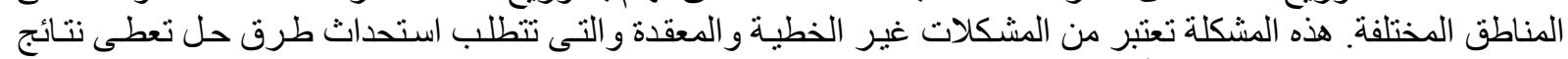

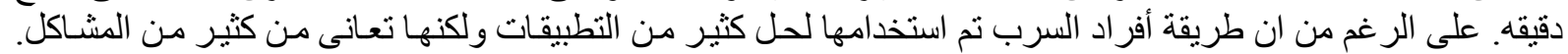

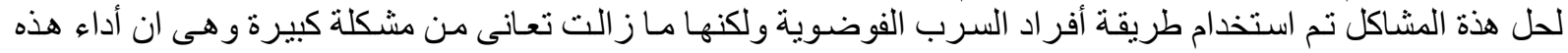

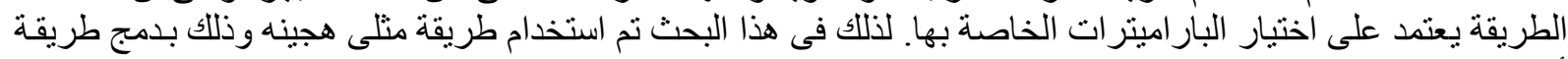
أفر اد السرب الفوضوية مع الخو ارزمية الجينية حيث يتم حساب القيمة المثلى للبار اميتر ات باستخدام لخوارزمية الجينية. يتم

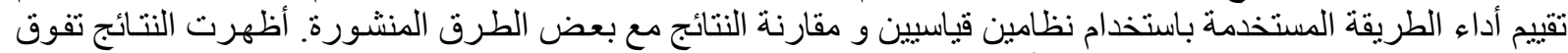

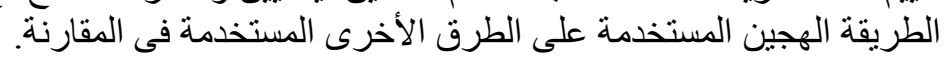

\begin{abstract}
Multi-area economic dispatch (MAED) deals with the optimal dispatch of multiple areas. MAED with tie line constraints, transmission losses, multiple fuel option and valve point effects is considered as a large scale non-linear optimization problem. An accurate optimization method to solve this problem is of great interest. However the conventional particle swarm optimization (PSO) has been applied to solve many optimization problems with success, it suffers from some drawbacks. Therefore, chaotic PSO (CPSO) has been used to treat these drawbacks. Choosing the parameters of CPSO has a great effect on its performance. So, a hybrid CPSO and genetic algorithm (HCPSOGA) method is employed in this paper to solve this problem. The hybrid method is derived by combining CPSO and GA where GA is used to optimize the parameters of CPSO. To show the effectiveness of the hybrid method, two test systems are used. The results show the superiority of the hybrid method over some published methods based on same test systems.
\end{abstract}

\section{Keywords}

Economic dispatch, multi area economic dispatch, multiple fuels, chaotic particle swarm optimization, genetic algorithm.

\section{Introduction}

Economic dispatch (ED) is one of the vital optimization problems in power system operation which aims to allocate the total load demand among the generation units while satisfying all constraints. ED problem is a complicated nonlinear optimization problem with several equality and inequality constraints. Many optimization methods were addressed in last few decades to solve single area ED problem [1-3].
In general, the generation units are divided into several generation areas which are interconnected by tie-lines. The main aim of multi-area economic dispatch (MAED) which is an extension of ED is determining the generation level and power transferred between areas in order to minimize the total fuel cost in all areas without violating any constraint. MAED is considered as a large scale non-linear optimization problem with several system and generators constraints [4]. 
In literature, various methods are used to solve MAED problem. In [4], Basu presented teaching-learning-based optimization algorithm for solving MAED problem. The MAED problem is solved with tie line constraints considering transmission losses, multiple fuels, and valve point effect and prohibited operating zones. Sudhaker et al. in [5] presented the differential evolution (DE) to solve MAED problem with tie line constraints. For small and medium sized MAED problems, the evolutionary programming (EP) method is presented in [6] to solve this problem. In [7] the MAED problem with multiple fuel option is solved using evolutionary programming Levenberg marquartdt method. Sharma et al. [8] have presented a classic PSO and DE methods to solve the reserve constrained multi-area economic dispatch problem with many constraints.

In modern power system operation, the total fuel cost function of generating units which supplied with multiple fuel sources may be segmented as piecewise quadratic cost functions for representing different fuel types [9]. The main aim of ED problem with multiple fuel options is to minimize total fuel cost among the available fuels (coal, oil or natural gas) for each unit satisfying all equality and inequality constraints. The ED problem with multiple fuel options is non-linear and non-convex problem. It contains the discontinuous values at each boundary forming multiple local optima. This makes the classical optimization methods are not suitable to solve this problem [9].

To solve ED problem with multiple fuel options, hierarchical techniques such as Hopfield neural network [9] and enhanced Lagrangian neural network [10] are used. These methods have some drawbacks where a large number of iterations are required to get the optimal solution and there is an oscillation during the transient process. Recently, the heuristic optimization methods such as genetic algorithm (GA) [11], particle swarm optimization (PSO) [12] and
Evolutionary programming method (EP) [13] have been applied with success.

However, the conventional PSO has been found to be robust in solving the ED problem, it suffers from some drawbacks. The performance of conventional PSO greatly depends on its parameters and it may be trapped in local optima so as to prematurely converge [14]. To overcome the drawbacks of the conventional PSO, chaotic PSO (CPSO) method is proposed by combining PSO with chaotic equation such as logistic equation [14-16]. In addition, some researchers combined it with GA and apply this hybrid method in many fields [1718].

In this paper, a hybrid chaotic particle swarm optimization and genetic algorithm (HCPSOGA) is employed to solve the multi area economic dispatch with multiple fuels. The hybrid method can be derived by combining CPSO and GA where the GA is used to optimize the parameters of CPSO which affects its performance. The employed method is evaluated using different test systems and compared with some published methods employing the same data.

The paper is organized as follows: Section 2 reviews the mathematical formulation of multi area economic dispatch with multiple fuels problem. Section 3 describes the HCPSOGA method. Experimental results and comparisons with other methods are presented in Section 4. Finally, Section 5 concludes the work.

\section{Problem Formulation}

The objective of MAED is to minimize the total generation cost of all areas with satisfying different constraints including tie-line capacity constraints [4, 11]. This work considers the MAED problem with valve point effect, multiple fuel options and transmission losses.

\subsection{Objective function}

The objective function $F$ is the total fuel cost of generating units of all areas and it can be defined as: 


$$
\begin{aligned}
F & =\sum_{i=1}^{N} \sum_{j=1}^{M_{i}} F_{i j}\left(P_{i j}\right) \\
& =\sum_{i=1}^{N} \sum_{j=1}^{M_{i}}\left(a_{i j}+b_{i j} P_{i j}+c_{i j} P_{i j}^{2}\right)
\end{aligned}
$$

where $F_{i j}\left(P_{i j)}\right.$ is the fuel cost of generator $j$ in area $i, P_{i j}$ is the power generated by generator $j$ in area $i, a_{i j}, b_{i j}$ and $c_{i j}$ are the cost coefficients of generator $j$ in area $i, N$ is the number of areas and $M_{i}$ is the number of the generators of area $i$.

By considering valve-point effects, the fuel cost of the generation unit can be defined by adding sinusoidal term as in (2):

$$
\begin{aligned}
& F=\sum_{i=1}^{N} \sum_{j=1}^{M i} F_{i j}\left(P_{i j}\right)= \\
& \sum_{i=1}^{N} \sum_{j=1}^{M i}\left[a_{i j}+b_{i j} P_{i j}+c_{i j} P_{i j}^{2}+\left|e_{i j} \times \sin \left(h_{i j}\left(P_{i j}^{\min }-P_{i j}\right)\right)\right|\right]
\end{aligned}
$$

Where, $e_{i j}, \quad h_{i j}$ are the valve-point coefficients of generator $j$ in area $i$ and $P_{i j}{ }^{\text {min }}$ is the minimum capacity limit of generator $j$ in area $i$.

By considering multiple fuel options, the fuel cost of the generation unit can be defined as follows:

$$
F=\sum_{i=1}^{N} \sum_{j=1}^{M i} F_{i j}\left(P_{i j}\right)
$$

Where $F_{i j}\left(P_{i j)}\right.$ can be defined using (4), in which $a_{i j k}, \quad b_{i j k}$ and $c_{i j k}$ are the cost coefficients of generator $j$ in area $i$ using the fuel type $k$.

\subsection{Constraints}

The MAED problem with multiple fuel options, valve point effects and transmission losses is subjected to the following constraints.

\subsubsection{Power balance constraint}

$$
\sum_{j=1}^{M i} P_{i j}=P_{D i}+P_{L i}+\sum_{z, z \neq i} T_{i z}, i=1,2, \ldots, N
$$

Where, $P_{D i}$ is the total load demand in area $i, P_{L i}$ is the transmission losses in area $i$ and $T_{i z}$ is the tie line power transfer from area $i$ to area $z$. When power flows from area $i$ to area $z, T_{i z}$ will be positive while $T_{i z}$ is negative when power flows from area $z$ to area $i$. In this paper, system loss is calculated as a function of units' power production using Kron's loss formula known as B-matrix coefficients [19] as follows.

$$
P_{L i}=\sum_{l=1}^{M i} \sum_{j=1}^{M i} P_{i j} B_{i l j} P_{i l}+\sum_{j=1}^{M i} B_{i j 0} P_{i j}+B_{00 i}
$$

Where $B_{i l j}$ is the $l j^{\text {th }}$ element of the loss coefficient square matrix in area $i, B_{i j 0}$ is the $j^{\text {th }}$ element of the loss coefficient vector in area $i$ and $B_{00 i}$ is the loss coefficient constant in area $i$.

$$
F_{i j}\left(P_{i j}\right)=\left\{\begin{array}{c}
a_{i j 1}+b_{i j 1} P_{i j}+c_{i j 1} P_{i j}^{2}+\left|e_{i j 1} \times \sin \left(h_{i j 1}\left(P_{i j 1}^{\min }-P_{i j}\right)\right)\right| \text { for fuel 1, } P_{i j}^{\text {min }} \leq P_{i j} \leq P_{i j 1} \\
a_{i j 2}+b_{i j 2} P_{i j}+c_{i j 2} P_{i j}^{2}+\left|e_{i j 2} \times \sin \left(h_{i j 2}\left(P_{i j 2}^{\min }-P_{i j}\right)\right)\right| \text { for fuel } 2, P_{i j 1} \leq P_{i j} \leq P_{i j 2} \\
\cdot \\
\cdot \\
a_{i j k}+b_{i j k} P_{i j}+c_{i j k} P_{i j}^{2}+\left|e_{i j k} \times \sin \left(h_{i j k}\left(P_{i j k}^{\min }-P_{i j}\right)\right)\right| \text { for fuel } k, P_{i j(k-1)} \leq P_{i j} \leq P_{i j}^{\max }
\end{array}\right.
$$

\subsubsection{Maximum and minimum limits of power generation:}

$$
P_{i j}^{\min } \leq P_{i j} \leq P_{i j}^{\max }
$$

Where $i=1,2, \ldots, N \quad$ and $j=1,2, \ldots, M i$

\subsubsection{Tie line capacity constraints}

The tie line power transfer $T_{i z}$ from area $i$ to area $z$ should not exceed the tie line transfer capacity for security consideration.

$$
-T_{i z}^{\max } \leq T_{i z} \leq T_{i z}^{\max }
$$


Where $T_{i z}^{\max }$ is the power flow limit from area $i$ to area $z$ and $-T_{i z}^{\max }$ is the power flow limit from area $z$ to area $i$.

\section{Hybrid Chaotic Particle Swarm Optimization and Genetic Algorithm}

\subsection{Particle Swarm Optimization (PSO)}

Particle swarm optimization (PSO) is a population based optimization technique developed by Kennedy and Eberhart in 1995 [20]. PSO begins with initial population of random solution. Each solution called particle which flies around in the search space to find the best solution. However PSO shares some features with GA, it has no crossover or mutation operators as in GA.

In PSO, every particle modifies its position according to its own experience and the experience of neighboring particles. The swarm direction of each particle is determined by the history of this particle and the experience of neighboring particles [14].

In n-dimensional search space, the updated velocity and position of each particle of PSO can be determined as follows [20]:

$$
\begin{gathered}
v_{i}^{k+1}=w \cdot v_{i}^{k}+c_{1} \cdot r_{1} \cdot\left(\text { pbest }_{i}^{k}-x_{i}^{k}\right) \\
+c_{2} \cdot r_{2} \cdot\left(\text { gbest }_{i}^{k}-x_{i}^{k}\right) \\
x_{i}^{k+1}=x_{i}^{k}+v_{i}^{k+1}
\end{gathered}
$$

where $x_{i}^{k}=\left(x_{i 1}^{k}, \ldots, x_{i n}^{k}\right)$ is the position of particle $i$ at iteration $k, v_{i}^{k}=\left(v_{i 1}^{k}, \ldots, v_{i n}^{k}\right)$ is the velocity of particle $i$ at iteration $k$, pbest $_{i}^{k}$ is the best previous position of particle $i$ at iteration $k$, gbest ${ }_{i}^{k}$ is the best position among all particles in the population, $w$ is weight parameter, $r_{1}, r_{2}$ are random numbers between 0 and 1 and $c_{1}, c_{2}$ are acceleration coefficients.
The general particle swarm optimization algorithm may be applied to any optimization problem. The steps of the conventional PSO algorithm are shown in Fig. 1.

Although the conventional PSO method has some advantages, it suffers from some drawbacks. It can be trapped in local optima so as to prematurely converge. This is due to that the performance of conventional PSO greatly depends on its parameters [14].

\subsection{Hybrid Algorithm}

In order to overcome the drawbacks of conventional PSO, a chaotic particle swarm optimization (CPSO) method is proposed in [15] by combining PSO with adaptive weight factor and logistic equation. The adaptive weight factor can be defined as follows [16]:

$$
w=\left\{\begin{array}{l}
w_{\text {min }}-\frac{\left(w_{\text {max }}-w_{\text {min }}\right)\left(f-f_{\text {min }}\right)}{f_{\text {avg }}-f_{\text {min }}}, f \leq f_{\text {avg }} \\
w_{\text {max }}, \quad f>f_{\text {avg }}
\end{array}\right.
$$

Where $w_{\min }$ and $w_{\max }$ is the minimum and maximum value of $w$, respectively, $f$ is the current objective value of the particle, $f_{\text {avg }}$ is the average objective value of all particles in the population and $f_{\min }$ is the minimum objective value of all particles in the population. 


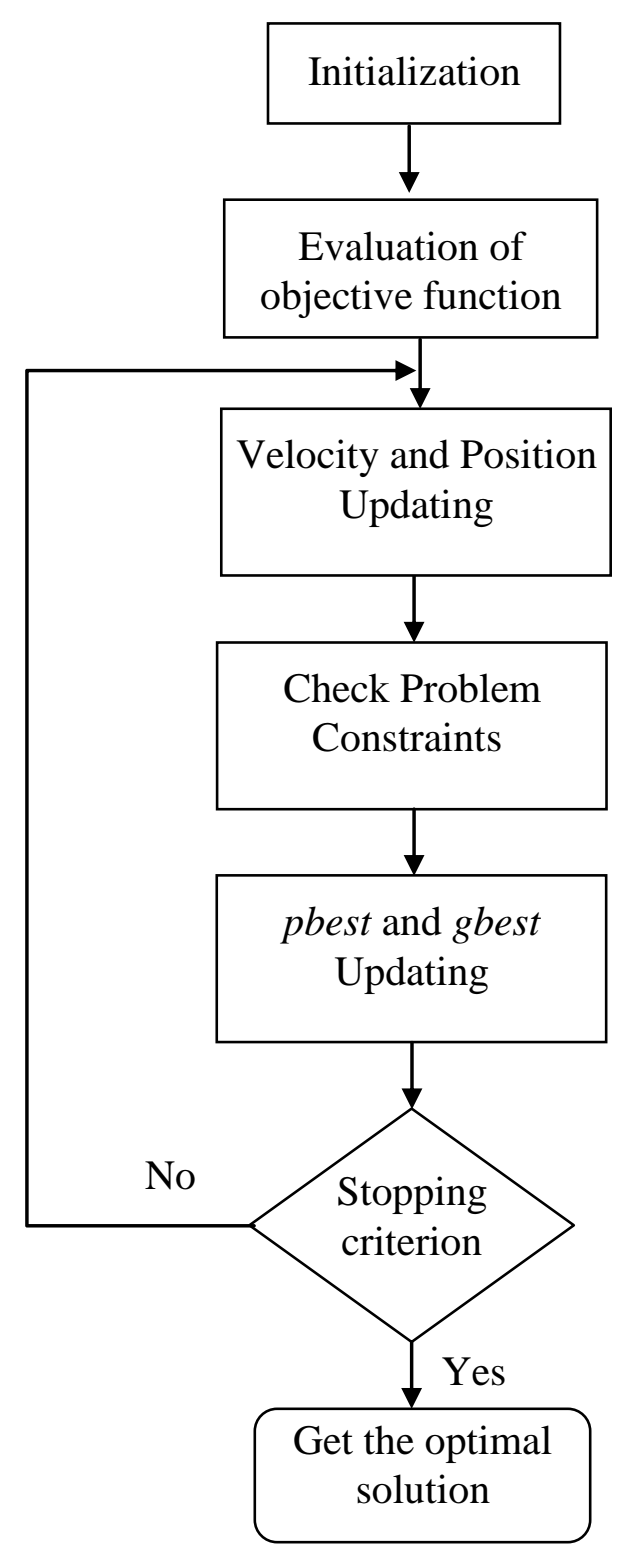

Fig. 1 Flowchart of Conventional PSO algorithm

To overcome the problem of trapping in local optimum in CPSO, chaos disturbance is used to jump out of the local optimum based on logistic equation in chaotic local search process as follows [14-16]:

$z x_{i}^{k+1}=4 z x_{i}^{k}\left(1-z x_{i}^{k}\right), \quad i=1,2, \ldots, n$

Where $z x_{i}^{k}$ is the $i^{\text {th }}$ chaotic variable at iteration $k, z x_{i}^{k}$ is distributed in the interval $(0,1.0)$ under the conditions that the initial $z x_{i}^{0} \in[0,1]$ and that $z x_{i}^{0} \notin[0.25,0.5,0.75]$ as in [14]. The chaotic variable $z x_{i}^{k}$ can be defined using the following equation [14]:

$$
z x_{i}^{k}=\frac{x_{i}^{k}-x_{\min , i}}{x_{\max , i}-x_{\min , i}}, \quad i=1,2, \ldots, n
$$

Then the decision variable $x_{i}^{k+1}$ can be calculated using this chaotic variable as follows [14]:

$$
x_{i}^{k+1}=x_{\min , i}+z x_{i}^{k+1}\left(x_{\max , i}-x_{\min , i}\right), \quad i=1,2, \ldots, n
$$

Based on the adaptive weight factor and logistic equation, the CPSO's procedures can be summarized as following [14-16]:

1) Randomly initialize the position and velocity of each particle in the population.

2) Calculate the fitness values of every particle. Then save the pbest $t_{i}^{k}$, gbest $_{i}^{k}$.

3) Use equations (9) and (10) to determine the position and velocity for the next iteration.

4) Update the weight using equation (11). Then calculate the objective values of all particles in the population and save some of the best solutions.

5) Execute chaotic local search based logistic equation using equations (12)(14)

6) Update both of pbest $_{i}^{k}$ and $g b e s t_{i}^{k}$.

7) Check if the maximum number of iterations is reached. If yes, save the best solutions. Otherwise, let increase the iteration number by 1 and go to Step 2 .

The performance of conventional PSO greatly depends on its parameters. So, selecting these parameters is a very important step in the PSO method. To overcome the drawbacks of conventional PSO and to choose the best value of these parameters in this work, a hybrid chaotic particle swarm optimization and genetic algorithm (HCPSOGA) is introduced. The HCPSOGA method can be derived by combining the CPSO [14-16] and GA where the GA is used to optimize the parameters of CPSO. These parameters are the acceleration factors $\left(c_{1}\right.$ and $\left.c_{2}\right)$ and minimum and maximum values of weigh factor $\left(w_{\min }\right.$ and $\left.w_{\max }\right)$. 
The steps of HCPSOGA method can be summarized as following:

\section{Step 1: Initialize the parameter:}

In this paper, the real value GA is used where real values of the parameters can be used directly to from each individual (chromosome). The acceleration and weight factors are randomly initialized to generate the chromosome. Each chromosome $(\mathrm{C}=$ $\left.\left\{c_{1}, c_{2}, w_{\min }, w_{\max }\right\}\right)$ represents the optimal value of the parameters of CPSO.

\section{Step 2: For each chromosome:}

Step 2.1: Generate the initial particle swarm:

Initialize the position and velocity of each particle in the population.

Step 2.2: Evaluation of each particle:

Calculate the fitness values of every particle. Then save the pbest ${ }_{i}^{k}$, gbest $_{i}^{k}$

Step 2.3: Update the velocity and position of each particle:

Use equations (9) and (10) to determine the position and velocity for the next iteration.

Step 2.4: Apply adaptive weight factor:

Update the weight using equation (11). Then calculate the objective values of all particles in the population and save some of the best solutions.

Step 2.5: Apply logistic equation:

Execute chaotic local search based logistic equation using equations (12)(14). Then update both of pbest $_{i}^{k}$ and gbest $_{i}^{k}$.

Step 2.6: Check the stopping criterion of CPSO:

In this paper, a predetermined maximum number of generations $\left(\mathrm{G}_{\text {max_PSO}}\right)$ is used as a termination condition. If the maximum number of generations is not reached, steps 2.2 to 2.5 can be repeated until the stopping criterion is satisfied.

\section{Step 3: Selection:}

A standard roulette wheel selection method is employed to select the fittest chromosomes from the current population.

\section{Step 4: Apply GA crossover:}

The crossover operator is used to produce two offspring from two parents chosen by roulette wheel selection method. The line arithmetical crossover is used as described in [21].

\section{Step 5: Conduct GA mutation:}

The mutation operation can contribute effectively to the diversity of the population. The Gaussian mutation has been used in this work as described in [21].

\section{Step 6: Elitist strategy:}

The chromosome that has the worst fitness value in the current generation is replaced by the chromosome that has the best fitness value in the old generation.

\section{Step 7: Check the stopping criterion of} GA:

In this paper, a predetermined maximum number of generations $\left(\mathrm{G}_{\text {max }_{\mathrm{GA}}}\right)$ is used as a termination condition. If the maximum number of generations is not reached, the steps 2 to 6 can be repeated using the chromosomes in the new generation until the stopping criterion is satisfied.

\section{Step 8: After the termination condition is satisfied:}

The chromosome which gives the best performance in the last generation is selected as the optimal values of CPSO's parameters and the corresponding fitness value is considered as the optimal solution of the MAED problem.

\section{Numerical Results}

Different test systems are used to show the effectiveness of the HCPSOGA method. In the implementation of HCPSOGA method, some parameters should be selected. The selection of suitable values of these parameters is very important in improving the speed of convergence and solution's quality. The parameters of CPSO $\left(c_{1}, c_{2}, w_{\min }\right.$ and $\left.w_{\max }\right)$ are optimized using GA for each test system. While the best value of other parameters for each system were selected from empirical tests by 
running the algorithm several times with different parameters combinations. The HABFGA method is implemented in Pentium 4 personal computer with $2.8 \mathrm{GHz}$ clock frequency and $2 \mathrm{~GB}$ of random access memory using MATLAB R2012a.

There are two different test cases are considered in this work. They are:

- $\quad$ Test system 1: This system consists of 10 generators divided into 3 areas. The valve-point effect, multi-fuel sources with 3 fuel options and transmission loss are considered in this system.

- $\quad$ Test system 2: This system consists of 40 generators divided into 4 areas. To be able to compare our results with other published methods, the valvepoint effect is considered while multifuel sources and transmission loss are not considered in this system.

\subsection{Test System 1}

This system has 10 generation units divided into 3 areas. The first area consists of the first 4 generators. The second area consists of the next 3 generators while the third area contains the last 3 generators. The data of this system can be found in [11]. While the B-coefficients data can be found in [4]. The GA parameters are population size $=100$, number of generation $=200$, crossover probability $=0.8$ and mutation of probability $=0.1$. The parameters of CPSO are number of particles in the swarm $=100$ and number of iteration $=200$.

In this case, the performance of HCPSOGA method is compared with teaching learning-based optimization (TLBO) [4], differential evolution (DE) [4], evolutionary programming (EP) [4] and real coded genetic algorithm (RCGA) [4] methods to show the effectiveness of the presented method. For the sake of fair comparison with other methods valve-point effect, multi-fuel sources with 3 fuel options and transmission loss are considered. Also, the load demand in the first area is assumed to be $50 \%$ of the total load demand $(2700$ MW), while the remaining demand is shared equally between the second and third areas (25\% for each area). Table 1 shows the power flow limit between different areas. Tables 2 and 3 show the results obtained from HCPSOGA, TLBO, DE, EP and RCGA methods. The results of the published methods used in this comparison have been directly quoted from their corresponding reference [4]. The cost convergence characteristic of HCPSOGA method is shown in Fig. 2.

Table 1, Power Flow Limit between Different Areas in Test System 1.

\begin{tabular}{|c|c|c|}
\hline \multicolumn{2}{|c|}{ Area } & Power flow \\
\hline From & To & MW \\
\hline 1 & 2 & 100 \\
\hline 1 & 3 & 100 \\
\hline 2 & 1 & 100 \\
\hline 2 & 3 & 100 \\
\hline 3 & 1 & 100 \\
\hline 3 & 2 & 100 \\
\hline
\end{tabular}


Table 2, Simulation Results for Test System 1.

\begin{tabular}{|c|c|c|c|c|c|c|c|c|c|c|c|}
\hline \multirow{2}{*}{ Area } & \multirow{2}{*}{$\begin{array}{l}\text { Power } \\
\text { (MW) }\end{array}$} & \multirow{2}{*}{\multicolumn{2}{|c|}{$\begin{array}{c}\text { TLBO [4] } \\
\text { Fuel }\end{array}$}} & \multirow{2}{*}{\multicolumn{2}{|c|}{$\begin{array}{c}\text { DE [4] } \\
\text { Fuel }\end{array}$}} & \multirow{2}{*}{\multicolumn{2}{|c|}{$\begin{array}{c}\text { EP [4] } \\
\text { Fuel }\end{array}$}} & \multirow{2}{*}{\multicolumn{2}{|c|}{$\begin{array}{c}\text { RCGA [4] } \\
\text { Fuel }\end{array}$}} & \multirow{2}{*}{\multicolumn{2}{|c|}{$\frac{\text { HCPSOGA }}{\text { Fuel }}$}} \\
\hline & & & & & & & & & & & \\
\hline \multirow{4}{*}{$\begin{array}{c}\text { Area } \\
1\end{array}$} & $\mathbf{P}_{1}$ & 224.31 & 2 & 225.45 & 2 & 223.85 & 2 & 239.09 & 2 & 223.89 & 2 \\
\hline & $\mathbf{P}_{2}$ & 210.66 & 1 & 210.17 & 1 & 209.58 & 1 & 216.12 & 1 & 210.52 & 1 \\
\hline & $\mathbf{P}_{3}$ & 491.69 & 2 & 491.28 & 2 & 496.07 & 2 & 484.15 & 2 & 490.47 & 2 \\
\hline & $\mathbf{P}_{4}$ & 240.63 & 3 & 240.89 & 3 & 237.99 & 3 & 240.62 & 3 & 241.08 & 3 \\
\hline \multirow{3}{*}{$\begin{array}{c}\text { Area } \\
2\end{array}$} & $\mathbf{P}_{5}$ & 249.57 & 1 & 251.01 & 1 & 259.43 & 1 & 259.66 & $\mathbf{1}$ & 248.66 & 1 \\
\hline & $P_{6}$ & 235.89 & 3 & 238.86 & 3 & 228.94 & 3 & 219.91 & 3 & 236.24 & 3 \\
\hline & $\mathbf{P}_{7}$ & 263.74 & 1 & 264.09 & 1 & 264.11 & 1 & 254.51 & 1 & 263.68 & 1 \\
\hline \multirow{3}{*}{$\begin{array}{c}\text { Area } \\
\mathbf{3}\end{array}$} & $\mathbf{P}_{8}$ & 237.13 & 3 & 236.99 & 3 & 238.23 & 3 & 231.36 & 3 & 237.82 & 2 \\
\hline & $\mathbf{P}_{9}$ & 332.59 & 1 & 326.54 & 1 & 331.29 & 1 & 341.96 & 1 & 333.25 & 3 \\
\hline & $\mathbf{P}_{10}$ & 249.46 & 1 & 250.33 & 1 & 246.60 & 1 & 248.28 & 1 & 249.99 & 1 \\
\hline \multicolumn{2}{|c|}{$\mathbf{P}_{\text {Loss 1 }}(\mathrm{MW})$} & \multicolumn{2}{|c|}{17.30} & \multicolumn{2}{|c|}{17.27} & \multicolumn{2}{|c|}{17.49} & \multicolumn{2}{|c|}{17.03} & \multicolumn{2}{|c|}{17.24} \\
\hline \multicolumn{2}{|c|}{$\mathbf{P}_{\text {Loss 2 }}(\mathrm{MW})$} & \multicolumn{2}{|c|}{9.66} & \multicolumn{2}{|c|}{9.77} & \multicolumn{2}{|c|}{10.01} & \multicolumn{2}{|c|}{9.70} & \multicolumn{2}{|c|}{9.71} \\
\hline \multicolumn{2}{|c|}{$\mathbf{P}_{\text {Loss } 3}(\mathrm{MW})$} & \multicolumn{2}{|c|}{8.73} & \multicolumn{2}{|c|}{8.59} & \multicolumn{2}{|c|}{8.61} & \multicolumn{2}{|c|}{8.94} & \multicolumn{2}{|c|}{8.66} \\
\hline \multicolumn{2}{|c|}{$\begin{array}{l}\text { Total Loss } \\
\text { (MW) }\end{array}$} & \multicolumn{2}{|c|}{35.69} & \multicolumn{2}{|c|}{35.63} & \multicolumn{2}{|c|}{36.11} & \multicolumn{2}{|c|}{35.67} & \multicolumn{2}{|c|}{35.61} \\
\hline Cos & $(\$ / \mathbf{h})$ & 653.9 & & 654.1 & & 655. & & 657. & & 650.9 & \\
\hline TiI & e (s) & 61.6 & & 65.0 & & 78.0 & & 83.8 & & 103.7 & \\
\hline
\end{tabular}

Table 3, Tie Line Power Flow Of Test System 1.

\begin{tabular}{|c|c|c|c|c|c|c|}
\hline \multicolumn{2}{|c|}{ Tie-line flow } & \multicolumn{5}{c|}{ Tie-line power flow (MW) } \\
\hline From & To & TLBO [4] & DE [4] & EP [4] & RCGA [4] & HCPSOGA \\
\hline Area 2 & Area 1 & $\mathbf{1 0 0 . 0 0}$ & $\mathbf{9 9 . 4 7}$ & $\mathbf{1 0 0 . 0 0}$ & $\mathbf{9 3 . 1 7}$ & $\mathbf{1 0 0 . 0 0}$ \\
\hline Area 3 & Area 1 & $\mathbf{1 0 0 . 0 0}$ & $\mathbf{1 0 0 . 0 0}$ & $\mathbf{1 0 0 . 0 0}$ & $\mathbf{9 3 . 8 7}$ & $\mathbf{1 0 0 . 0 0}$ \\
\hline Area 3 & Area 2 & $\mathbf{3 5 . 4 6}$ & $\mathbf{3 0 . 2 8}$ & $\mathbf{3 2 . 5 2}$ & $\mathbf{4 3 . 7 8}$ & $\mathbf{3 6 . 0 4}$ \\
\hline
\end{tabular}

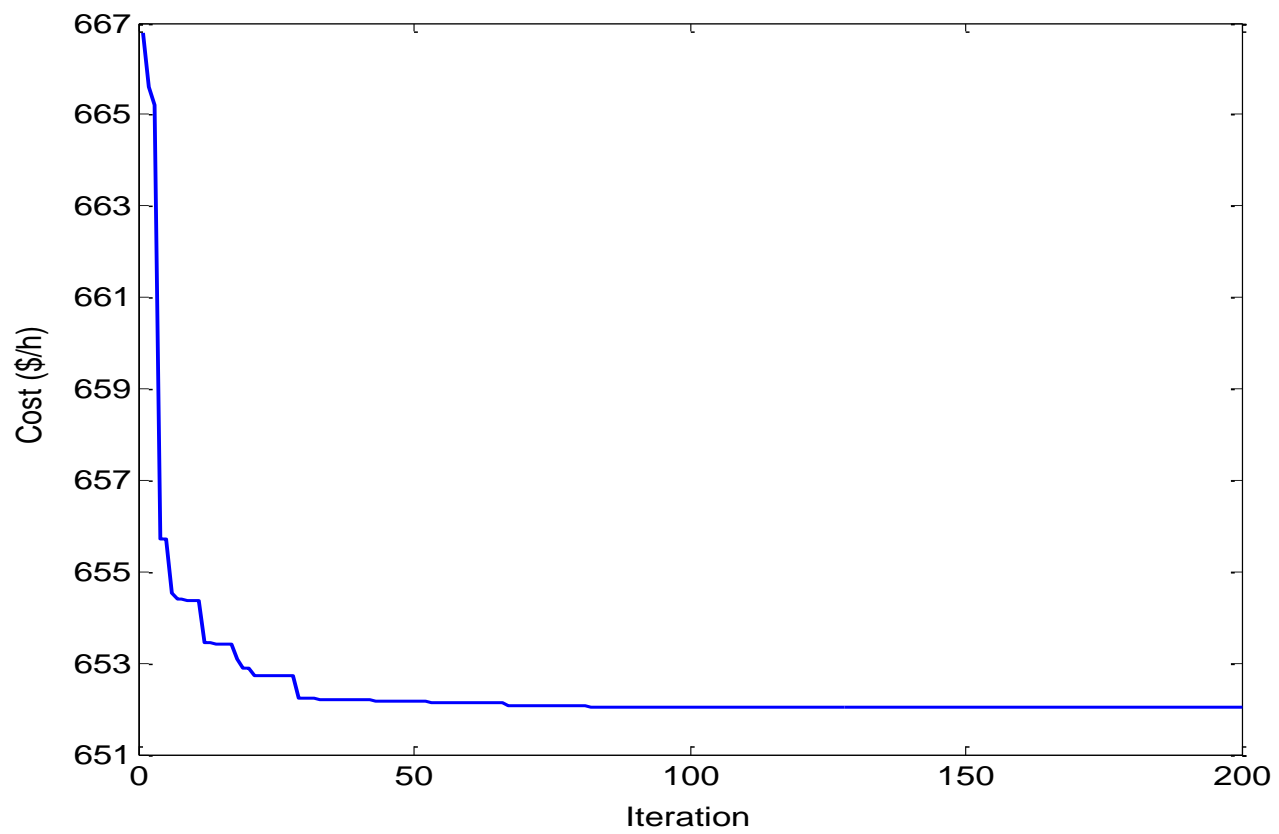

Fig. 2 Cost convergence characteristic of HCPSOGA method for test system 1

These results show the superiority of the HCPSOGA method over other published methods. It gives total costs less than those from other methods.

\subsection{Test System 2}

This system has 40 generation units divided equally into 4 areas (10 generators for each area) with total load demand 10500 
MW. The data of this system can be found in [22]. The GA parameters are population size $=100$, number of generation $=500$, crossover probability $=0.8$ and mutation of probability $=0.1$. The parameters of CPSO are number of particles in the swarm $=100$ and number of iteration $=500$.

In this case, the performance of HCPSOGA method is compared with artificial bee colony optimization (ABCO) [23] and DE [23] methods to show the effectiveness of the presented method. In all methods, the valve-point effect is considered while multi-fuel sources and transmission loss are not considered. Also, the maximum number of iteration is chosen to be 500. Fig. 3 shows the load demand of each area and power flow limit between different areas. Tables 4 and 5 show the results obtained from HCPSOGA, ABCO and DE methods. The results of the published methods used in this comparison have been directly quoted from their corresponding reference [23]. The cost convergence characteristic of HCPSOGA method is shown in Fig. 4.

Again, the results prove the superiority of the HCPSOGA method over other methods.

\subsection{Discussions}

To investigate the effectiveness of the HCPSOGA method, its performance is compared with some published. These methods are TLBO, DE, EP and RCGA methods for test system 1 and $\mathrm{ABCO}$ and DE for the test system 2 . The results of these published methods have been directly quoted from their corresponding references. From the above results, we can notice that the HCPSOGA significantly outperformed other methods used in comparisons for both test systems.
The HCPSOGA achieved a cost of $650.97 \$ / \mathrm{h}$ and $123531.2 \$ / \mathrm{h}$ for test system 1 and 2, respectively which is a yearly saving of about 26455 \$ and 4189032 \$ compared to the lowest cost obtained by other methods in test system 1 and 2 , respectively. Also, the efficiency of the HCPSOGA method is proved in the large non-convex type problem (test system 2).

Based on the above results, the HCPSOGA method has high-speed convergence, but its computational burden is high compared with other published method. The real life MAED problem is solved off line and solution time of several minutes is acceptable. This makes it possible to use the HCPSOGA method to solve the real life ED problem.

\section{Conclusions}

In this paper, a hybrid method called HCPSOGA was employed to solve the multi area ED problem with multiple fuel option. This hybrid method can be derived by combining PSO, adaptive weight factor, logistic equation and GA, so that the drawbacks of original PSO can be avoided. To show the feasibility and efficiency of the hybrid method, two commonly used standard test systems are used. The numerical results were compared with the recently reported approaches. The results revealed that the solution obtained by the presented method led to a smaller total generating cost than those obtained using other published methods. However the computational time of the presented method is higher than other methods, it is still acceptable for the real time applications for MAED problem. 


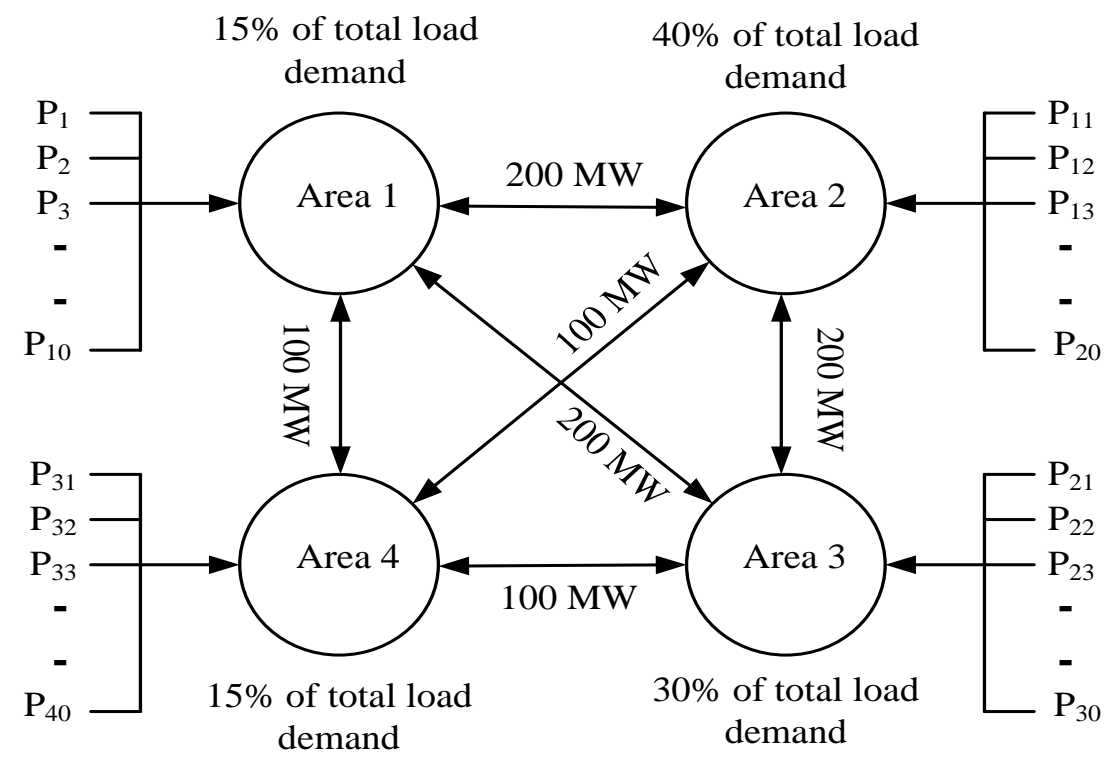

Fig. 3 Four area system (Test system 2)

Table 4, Simulation Results for Test System 2.

\begin{tabular}{|c|c|c|c|c|c|c|c|c|c|}
\hline Area & $\begin{array}{l}\text { Power } \\
(\mathrm{MW})\end{array}$ & $\begin{array}{c}\mathbf{A B C O} \\
{[23]}\end{array}$ & $\begin{array}{c}\text { DE } \\
{[23]}\end{array}$ & HCPSOGA & Area & $\begin{array}{l}\text { Power } \\
\text { (MW) }\end{array}$ & $\begin{array}{c}\mathbf{A B C O} \\
{[23]}\end{array}$ & DE [23] & HCPSOGA \\
\hline \multirow{10}{*}{ Area 1} & $\mathbf{P}_{1}$ & 111.10 & 93.08 & 115.01 & \multirow{10}{*}{$\begin{array}{c}\text { Area } \\
\mathbf{3}\end{array}$} & $\mathbf{P}_{21}$ & 527.19 & 547.63 & 525.97 \\
\hline & $\mathbf{P}_{2}$ & 109.98 & 109.06 & 110.18 & & $\mathbf{P}_{22}$ & 502.08 & 523.49 & 500.08 \\
\hline & $\mathbf{P}_{3}$ & 100.92 & 89.75 & 101.52 & & $\mathbf{P}_{23}$ & 530.37 & 522.63 & 532.34 \\
\hline & $\mathbf{P}_{4}$ & 190.00 & 116.95 & 195.20 & & $\mathbf{P}_{24}$ & 542.34 & 545.94 & 540.54 \\
\hline & $\mathbf{P}_{5}$ & 96.94 & 97.00 & 91.84 & & $\mathbf{P}_{25}$ & 520.25 & 523.66 & 519.21 \\
\hline & $\mathbf{P}_{6}$ & 96.97 & 140.00 & 93.90 & & $\mathbf{P}_{26}$ & 533.64 & 527.37 & 536.04 \\
\hline & $\mathbf{P}_{7}$ & 259.69 & 283.73 & 250.61 & & $\mathbf{P}_{27}$ & 10.00 & 10.00 & 10.00 \\
\hline & $\mathbf{P}_{8}$ & 276.87 & 286.27 & 275.37 & & $\mathbf{P}_{28}$ & 10.00 & 15.79 & 10.97 \\
\hline & $\mathbf{P}_{9}$ & 300.00 & 284.91 & 302.01 & & $\mathbf{P}_{29}$ & 10.00 & 10.00 & 10.00 \\
\hline & $\mathbf{P}_{10}$ & 130.69 & 131.64 & 130.19 & & $\mathbf{P}_{\mathbf{3 0}}$ & 96.77 & 93.03 & 95.37 \\
\hline \multirow{10}{*}{ Area 2} & $\mathbf{P}_{11}$ & 245.10 & 169.87 & 252.11 & \multirow{10}{*}{$\begin{array}{c}\text { Area } \\
4\end{array}$} & $\mathbf{P}_{31}$ & 190.00 & 190.00 & 190.00 \\
\hline & $\mathbf{P}_{12}$ & 94.00 & 110.97 & 92.33 & & $\mathbf{P}_{32}$ & 168.68 & 157.89 & 169.92 \\
\hline & $\mathbf{P}_{13}$ & 125.00 & 229.89 & 120.41 & & $\mathbf{P}_{33}$ & 173.62 & 190.00 & 170.61 \\
\hline & $\mathbf{P}_{14}$ & 434.81 & 387.47 & 436.51 & & $\mathbf{P}_{34}$ & 186.37 & 200.00 & 183.30 \\
\hline & $\mathbf{P}_{15}$ & 390.67 & 427.75 & 390.00 & & $\mathbf{P}_{35}$ & 200.00 & 90.00 & 200.00 \\
\hline & $\mathbf{P}_{16}$ & 395.00 & 478.28 & 391.22 & & $\mathbf{P}_{36}$ & 164.96 & 149.45 & 167.97 \\
\hline & $\mathbf{P}_{17}$ & 500.00 & 490.18 & 500.99 & & $\mathbf{P}_{37}$ & 92.56 & 110.00 & 90.06 \\
\hline & $\mathbf{P}_{18}$ & 500.00 & 490.95 & 500.92 & & $\mathbf{P}_{38}$ & 96.99 & 88.16 & 100.89 \\
\hline & $P_{19}$ & 530.79 & 511.92 & 531.72 & & $\mathbf{P}_{39}$ & 109.82 & 25.00 & 112.31 \\
\hline & $\mathbf{P}_{20}$ & 514.41 & 511.82 & 513.88 & & $\mathbf{P}_{40}$ & 431.40 & 538.47 & 438.50 \\
\hline \multicolumn{7}{|c|}{ Cost $(\$ / h)$} & 124009.4 & 124544.1 & 123531.2 \\
\hline \multicolumn{7}{|c|}{ Time (s) } & 126.93 & 134.81 & 190.58 \\
\hline
\end{tabular}


Table 5 Tie Line Power Flow Of Test System 2.

\begin{tabular}{|c|c|c|c|c|}
\hline \multicolumn{2}{|c|}{ Tie-line flow } & \multicolumn{3}{c|}{ Tie-line power flow (MW) } \\
\hline From & To & $\begin{array}{c}\text { ABCO } \\
{[23]}\end{array}$ & DE $[23]$ & HCPSOGA \\
\hline Area 1 & Area 2 & 191.71 & 200 & 190.99 \\
\hline Area 3 & Area 1 & $\mathbf{6 . 6 7}$ & $\mathbf{9 1 . 5 4}$ & $\mathbf{2 3 . 8 5}$ \\
\hline Area 3 & Area 2 & $\mathbf{1 8 3 . 1 9}$ & $\mathbf{1 4 7 . 8 9}$ & $\mathbf{1 8 1 . 3 1}$ \\
\hline Area 4 & Area 1 & $\mathbf{8 6 . 8 6}$ & $\mathbf{5 1 . 0 8}$ & $\mathbf{4 8 . 0 1}$ \\
\hline Area 4 & Area 2 & $\mathbf{9 5 . 3 2}$ & $\mathbf{4 2 . 9 9}$ & $\mathbf{9 0 . 7 2}$ \\
\hline Area 4 & Area 3 & $\mathbf{5 7 . 2 2}$ & $\mathbf{6 9 . 9 0}$ & $\mathbf{9 5 . 2 3}$ \\
\hline
\end{tabular}

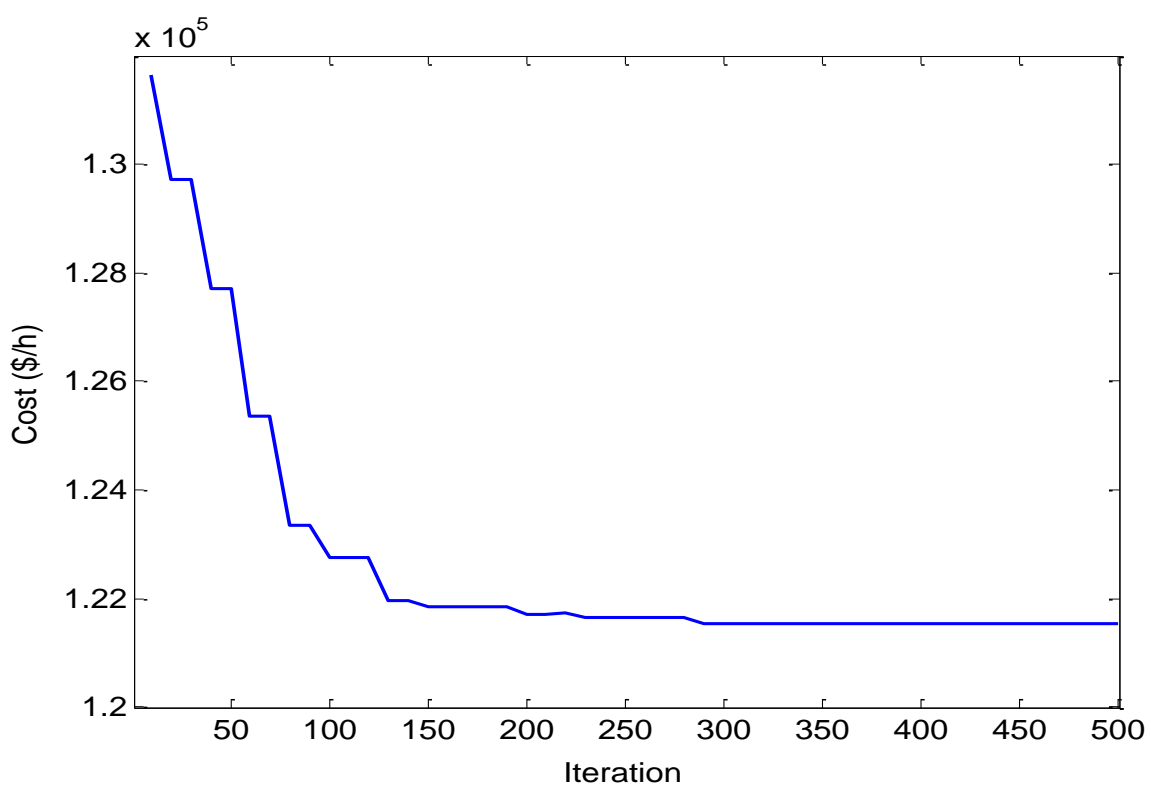

Fig. 4 Cost convergence characteristic of HCPSOGA method for test system 2.

\section{References}

[1.] Mandala B., Royb P. and Mandal S., "Economic Load Dispatch Using Krill Herd Algorithm", International Journal of Electrical Power Energy Systems, Vol. 57, pp. 1-10, 2014.

[2.] Wang M., Gooi H., Chen S. and Lu S. "A Mixed Integer Quadratic Programming for Dynamic Economic Dispatch with Valve Point Effect", IEEE Transaction on Power Systems, Vol. 29, no. 5, pp. 2097-2106, 2014.

[3.] Elattar E. "A Hybrid Genetic Algorithm and Bacterial Foraging Approach for Dynamic Economic Dispatch Problem", International Journal of Electrical Power Energy Systems, Vol. 69, pp. 18-26, 2015.

[4.] Basu, M., "Teaching-Learning-Based Optimization Algorithm for Multi-
Area Economic Dispatch", Energy, Vol. 68, pp. 21-28, 2014.

[5.] Sudhakar A., Chandram K. and Laxmi A., "Differential Evolution for Solving Multi Area Economic Dispatch", International Conference on Advances in Computing, Communications and Informatics (ICACCI'2014), Sep. 2427, 2014, pp. 1146-1151.

[6.] Jayabarathi T., Sadasivam G., and Ramachandran V., "Evolutionary Programming Based Multiarea Economic Dispatch with Tie Line Constraints", Electrical Machines and Power Systems, vol. 28, pp. 11651176, 2000.

[7.] Manoharam P., Kannan P. and Ramanathan V., "A Novel EP Approach for Multi-Area Economic Dispatch with Multiple Fuel Options", 
Turkey Journal of Electrical Engineering and Computer Science, Vol. 17, pp. 1-19, 2009.

[8.] Sharma M., Pandit M. and Srivastava L., "Reserve Constrained Multi-Area Economic Dispatch Employing Differential Evolution with Time varying Mutation", International Journal of Electrical Power Energy Systems, Vol. 33, pp. 753-766, 2011.

[9.] Vo D. and Ongsakul W., "Economic Dispatch with Multiple Fuel Types by Enhanced Augmented Lagrange Hopfield Network", Applied Energy, Vol. 91, pp. 281-289, 2012.

[10.] Lee S. and Kim Y., "An Enhanced Lagrangian Neural Network for the ELD Problems with Piecewise Quadratic Cost Functions and Nonlinear Constraints", Electric Power System Research, Vol. 60, pp. 167177, 2002.

[11.] Chiang C., "Improved Genetic Algorithm for Power Economic Dispatch of Units with Valve-Point Effects and Multiple Fuels", IEEE Transactions on Power Systems, Vol. 20, no. 4, pp.1690-1699, 2005.

[12.] Selvakumar A. and Thanushkodi K., "A New Particle Swarm Optimization Solution to Nonconvex Economic Dispatch Problems," IEEE Transactions on Power Systems, Vol. 22, no. 1, pp. 42-51, 2007.

[13.] Jayabarathi T., Sadasivam G. and Ramachandran V., "Evolutionary Programming Based Economic Dispatch of Generator with Prohibited Operating Zones," Electric Power System Research, Vol. 52, pp. 261266, 1999.

[14.] Jiejin C., Xiaoqian M., Lixiang L. and Haipeng P., "Chaotic Particle Swarm Optimization for Economic Dispatch Considering the Generator Constraints", Energy Conversion and Management, Vol. 48, pp. 645-653, 2007.

[15.] Liu B., Wang L., Jin Y. H., Tang F. and Huang D. X., "Improved Particle
Swarm Optimization Combined With Chaos", Solitons Fractals, Vol. 25, pp.1261-1271, 2005.

[16.] Zhu Y. and et. al., "Hierarchical Economic Load Dispatch Based on Chaotic-particle Swarm Optimization", Ninth International Conference on Natural Computation (ICNC), Jul. 23-25, 2013, pp. 517-521.

[17.] Soleimani H. and Kannan G.," A Hybrid Particle Swarm Optimization and Genetic Algorithm for Closedloop Supply Chain Network Design in Large-scale Networks", Applied Mathematical Modelling, Vol. 39, no. 14, pp. 3990-4012, 2015.

[18.] Wu J., Long J. and Liu M.," Evolving RBF Neural Networks for Rainfall Prediction Using Hybrid Particle Swarm Optimization and Genetic Algorithm", Neurocomputing, Vol. 148, pp. 136-142, 2015.

[19.] Abdelaziz A., Kamh M., Mekhamer S. and Badr M., "A Hybrid HNN-QP Approach for Dynamic Economic Dispatch Problem", Electric Power System Research, Vol. 78, pp. 17841788, 2008.

[20.] Kennedy J. and Eberhart R., "Particle Swarm Optimization", In Proc. IEEE Int. Conf. Neural Networks (ICNN'95), Vol. IV, Perth, Australia,1995, pp. 1942-1948.

[21.] Michalewicz Z., "Genetic Algorithms + Data Structures $=$ Evolving Programs", Springer-Verlag, Berlin Heidelberg, 1996.

[22.] Sinha N., Chakrabarti R. and Chattopadhyay P. K., "Evolutionary Programming Techniques for Economic Load Dispatch", IEEE Transactions on Evol. Comput., Vol, 7, pp. 83-94, 2003.

[23.] Basu M., "Artificial Bee Colony Optimization for Multi-area Economic Dispatch", International Journal of Electrical Power and Energy Systems, Vol. 49, pp. 181-187, 2013. 